\title{
A susceptibility locus rs7099208 is associated with non-obstructive azoospermia via reduction in the expression of FAM160B1
}

\author{
Yan Zhang ${ }^{1,4}$, Jing Qian ${ }^{1,2}$, Minghui $\mathrm{Wu}^{1,2}$, Mingxi Liu ${ }^{1,2}$, Kai Zhang ${ }^{1,3}$, Yuan Lin $^{1,3}$, Xuejiang Guo ${ }^{1,2}$, \\ Zuomin Zhou ${ }^{1,2}$, Zhibin $\mathrm{Hu}^{1,3}$, Jiahao Sha ${ }^{1,2, 凶}$ \\ ${ }^{1}$ State Key Laboratory of Reproductive Medicine $;{ }^{2}$ Department of Histology and Embryology; ${ }^{3}$ Department of Epidemiology and \\ Biostatistics and Key Laboratory of Modern Toxicology of Ministry of Education Nanjing Medical University, Nanjing, Jiangsu \\ 210029, China; \\ ${ }^{4}$ The Center for Reproductive Medicine, The Second Affiliated Hospital of Nanjing Medical University, Nanjing, Jiangsu \\ 210011, China.
}

\begin{abstract}
Non-obstructive azoospermia (NOA) is a severe defect in male reproductive health that occurs in $1 \%$ of adult men. In a previous study, we identified that rs7099208 is located within the last intron of FAMI60B1 at 10q25.3. In this study, we analysed expression Quantitative Trait Loci (eQTL) of FAM160B1, ABLIM1 and TRUB1, the three genes surrounding rs7099208. Only the expression level of FAM160B1 was reduced for the homozygous alternate genotype (GG) of rs7099208, but not for the homozygous reference or heterozygous genotypes. FAM160B1 is predominantly expressed in human testes, where it is found in spermatocytes and round spermatids. From 17 patients with NOA and five with obstructive azoospermia (OA), immunohistochemistry revealed that expression of $F A M 160 B 1$ is reduced, or undetectable in NOA patients, but not in OA cases or normal men. We conclude that rs7099208 is associated with NOA via a reduction in the expression of FAMI60B1.
\end{abstract}

Keywords: non-obstructive azoospermia, obstructive azoospermia, rs7099208, FAM160B1, expression Quantitative Trait Loci, apoptosis

\section{Introduction}

Approximately $15 \%-20 \%$ of couples are infertile, with males and females suffering equally from infertility ${ }^{[1]}$. Non-obstructive azoospermia (NOA), a severe defect in male reproductive health, occurs in $1 \%$ of adult men $^{[2]}$. It has been reported that a defect in the AZF region of the Y chromosome causes 10\%-15\% of idiopathic $\mathrm{NOA}^{[3]}$. However, other genetic causes of NOA remain unclear. Many gene knockouts lead to NOA in mice, but few corresponding defects have been observed in NOA men.

\footnotetext{
${ }^{\square}$ Corresponding authors: Dr. Jiahao Sha, State Key Laboratory of Reproductive Medicine, Department of Histology and Embryology, Nanjing Medical University, Nanjing, Jiangsu 210029, China. Tel/fax: +86-25-86862908/+86-25-86862908, E-mail: shajh@njmu.edu.cn.
}

One explanation of the relationship between risk loci and disease is that these loci are associated with mRNA expression of relevant genes ${ }^{[4-5]}$. Protein markers for disease also could have been discovered by GWAS analysis ${ }^{[6-7]}$. To investigate genetic variation in NOA patients, we recently conducted a genome-wide association study (GWAS) of NOA in Han Chinese based on genotyping 587,347 single nucleotide polymorphisms (SNPs) in 981 NOA cases and 1,657 controls $^{[8]}$. We tested promising associations in an extended three-stage validation using 3,608 NOA cases and 5,909 controls. In the combined analysis, meta-analysis

Received 28 February 2015, Revised 08 March 2015, Accepted 06 May 2015, Epub 08 August 2015

CLC number: $\mathrm{R}_{698}{ }^{+} .2$, Document code: A

The authors reported no conflict of interests. 
was used to combine the results of GWAS scan and three validations. We identified three risk loci associated with NOA (rs7194 at 6p21.32, rs7099208 at 10q25.3, and rs 13206743 at $6 \mathrm{pl} 2.2)^{[9]}$. According to our previous study, genotype of rs7099208 in NOA patients is 43/943/3552 (Variant homozygote/Heterozygote/Wild type) and in control population is 35/1209/6312 (Variant homozygote/ Heterozygote/Wild type), and minor allele frequency (MAF) of rs7099208 is 0.11 in NOA patients, which is higher than 0.08 in control population, $P=6.41 \times 10^{-14[9]}$. However, it is still unknown whether rs7099208 affects mRNA expression.

In this work, we tested the hypothesis that expression of risk loci related genes is altered in testes from NOA patients. Our approach examined samples from patients with NOA and obstructive azoospermia $(\mathrm{OA})$, and the germ cell line of male mice.

\section{Materials and methods}

\section{Expression Quantitative Trait Loci (eQTL)}

The publicly available RNA-seq and genotyping data of 53 normal testis samples and 167 whole blood samples from the Genotype-Tissue Expression project (GTEx, http://commonfund.nih.gov/GTEx/index) were used to assess Gene Expression Quantitative Trait Loci (eQTL) for mRNA expression of FAM160Bl and genes neighbouring SNP rs7099208 ${ }^{[10]}$.

\section{Patients}

This study was approved by the institutional review boards of each participating institution. Participants were solicited from individuals attending the First Affiliated Hospital Reproductive Centre of Nanjing Medical University for knockdown of infertility. Controls $(n=7)$ were defined as individuals who were diagnosed with normal spermatogenesis or OA. Patients diagnosed with NOA were the experimental group $(n=17)$. Six of the 17 formed the spermatid arrest sample, and the others were the spermatocyte arrest sample. For sample specific information see Supplementary Material.

\section{Processing of testicular biopsy material}

This study was approved by the ethics committees of Nanjing Medical University and was conducted in accordance with national and international guidelines. A testicular biopsy was performed in all samples $(n=24)$. A small incision was made in the tunica albuginea to remove tissue samples, which comprised about $3 \mathrm{~mm}^{3}$ in total.

\section{Indirect immunohistochemistry and immunofluorescence}

Indirect immunohistochemistry and immunofluorescence were performed as previously described ${ }^{[11-13]}$. To prepare sections, tissue from normal testes was fixed in Bouin fluid and dehydrated through a series of graded alcohols. The testes were then embedded in paraffin at $65^{\circ} \mathrm{C}$ and sections were cut for immunohistochemistry. The testis sections were hydrated with series of graded alcohols. After endogenous peroxidase activity was blocked with $3 \% \mathrm{H}_{2} \mathrm{O}_{2}$, non-specific binding was blocked with $10 \%$ normal goat serum. The sections were incubated overnight at $4{ }^{\circ} \mathrm{C}$ with primary antibody against FAM160B1 (Abgent) at a dilution of 1:100. After extensive washes in PBS, the sections were incubated with the secondary antibody for 1 hour at $37^{\circ} \mathrm{C}$. Next, DBA colour liquid was added to the sections and the reaction product was generated. After immunostaining, the testis sections were counterstained with hematoxylin and examined under a light microscope. For immunofluorescence, GC2 cells were fixed with $4 \%$ PFA at room temperature and $70 \%$ Ethanol at $-20{ }^{\circ} \mathrm{C}$. Cells were analysed using an epifluorescence microscope (Zeiss 710) at room temperature and Image Pro-Plus version software (Carl Zeiss, Germany) was used for analysis and imaging. Images were acquired using a $100 \times$ magnification oil lens (Zeiss). In brief, green fluorescence was excited with a 488 $\mathrm{nm}$ diode laser and red fluorescence with a $555 \mathrm{~nm}$ diode laser. Multicolour images were acquired sequentially.

\section{PCR}

Total RNA was extracted from cells with moFAM160B1 and mo-control tissue using an RNeasy plus Micro Kit (QIAGEN, 74034). RNA was reversetranscribed into cDNA using a PrimeScript RT reagent Kit (Takara, DRR037S). PCR was performed with GoTaq Green Master Mix (Promega, M7122), and real-time PCR was performed with SYBR Premix EX Taq II (Takara, DRR081B). In PCR, the forward and reverse primers used were: MUS (F) GAGGGCTTGA TGCTCTTGGT and (R) GCTGTATGAGTCCAACC CCC; HUM (F) GGCTGCAAAGTGCCTTACAC and (R) CGAACGATGCGATGAAGCAG.

The PCR started at $95{ }^{\circ} \mathrm{C}$ for 30 seconds and was performed as follows: 28 cycles of denaturation at $95{ }^{\circ} \mathrm{C}$ for 5 seconds, annealing at $60{ }^{\circ} \mathrm{C}$ for 30 seconds and elongation at $72{ }^{\circ} \mathrm{C}$ for 30 seconds.

\section{Western blotting assay}

Samples containing $20 \mu \mathrm{g}$ of protein (measured by the Bradford method) were used for Western blotting. 
Proteins from mo-FAM160Bl cells and control samples were subject to electrophoresis on a $10 \%$ sodium dodecyl sulfate polyacrylamide gel. The proteins were then transferred from the gel onto a nitrocellulose membrane (GE Healthcare Bio-Sciences AB, Uppsala, Sweden). Membranes were blocked in a solution of phosphate-buffered saline (PBS) containing 5\% non-fat milk powder for 1 hour, and then incubated overnight at $4{ }^{\circ} \mathrm{C}$ with the following primary antibodies: anti- FAM160B1 (1:1,000; Abgent) and anti- $\beta$-tubulin (1:2,000; Abcam). After four 10 minutes washes in PBS, the membranes were incubated for 1 hour at $37^{\circ} \mathrm{C}$ with horseradish peroxidase-conjugated secondary antibody. Specific proteins were detected using an ECL kit and AlphaImager (FluorChem5500; Alpha Innotech, San Leandro, CA).

\section{Cell culture and morpholino knockdown}

The mouse spermatocyte cell line GC2-spd (ATCC catalogue number CRL-2196, Manassas, VA, USA) was used for in vitro studies. Growth media contained $10 \%$ foetal bovine serum (GIBCO) and 5\% penicillin/ streptomycin, and was maintained in a $37{ }^{\circ} \mathrm{C}$ incubator in a humidified, $5 \% \mathrm{CO}_{2}$ atmosphere. The knockdown
A

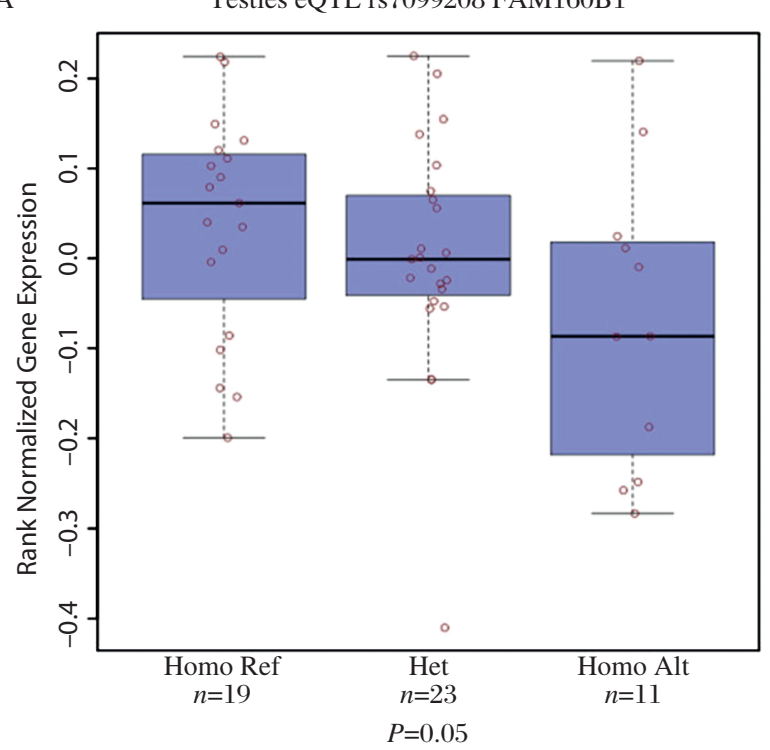

$\mathrm{C}$

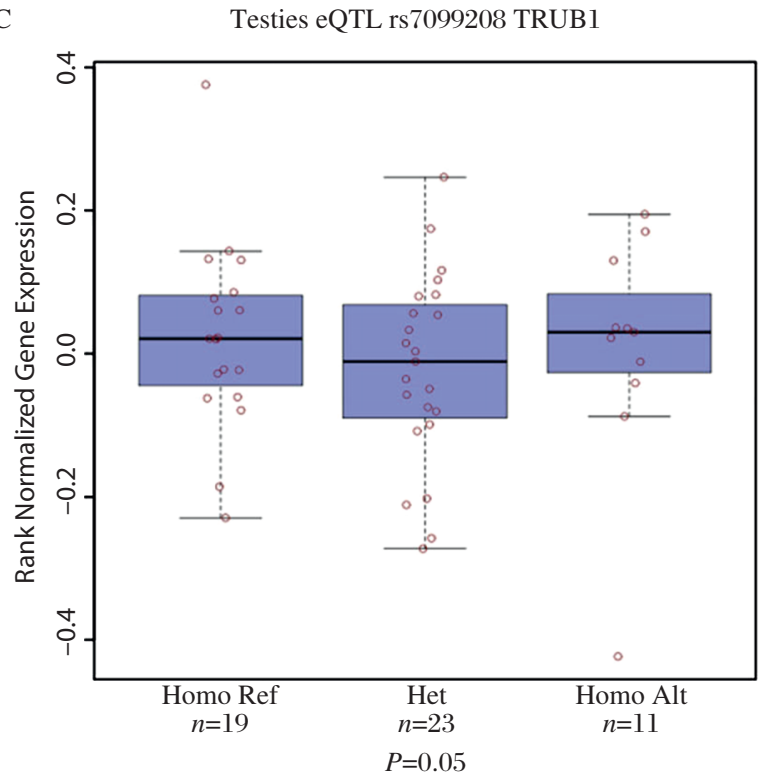

B

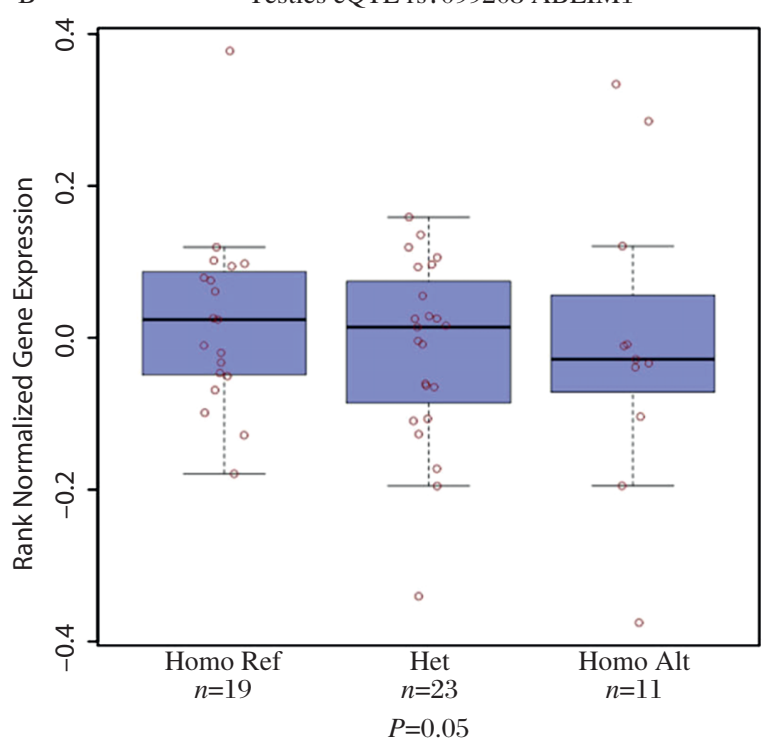

$\mathrm{D}$

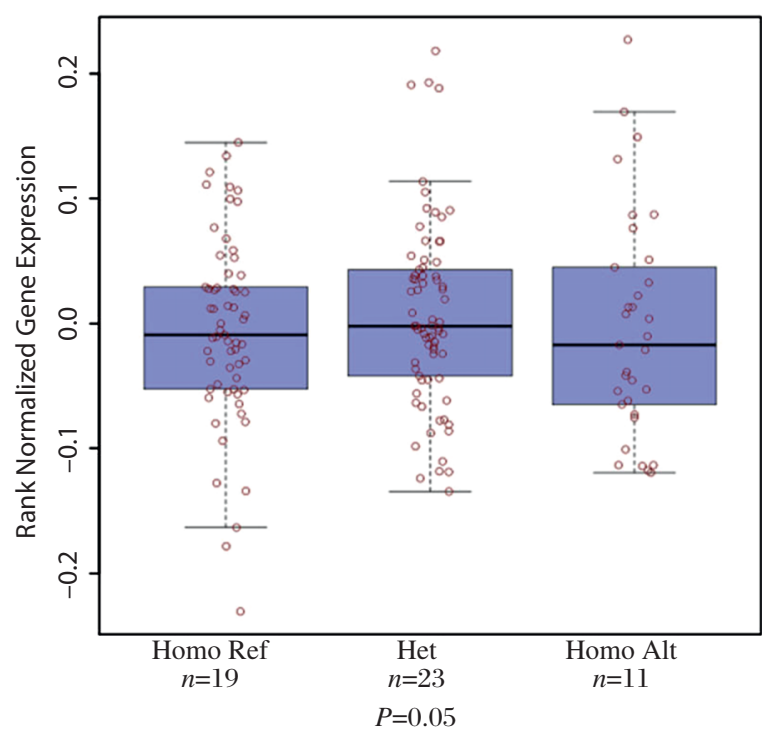

Fig. 1 Through eQTL analysis SNP rs7099208 with gene from the Genotype-Tissue Expression project (GTEx, http:// commonfund.nih.gov/GTEx/index). SNP rs7099208 existed eQTL with gene FAM160B1(A), P=0.05, instead of the other two: ABLIMI (B) and TRUBI (C). D: This eQTL was tissue-specific, existed only in the testis, while outside of comprehensive blood. 
was performed using morpholinos (splice blocking and translation blocking). Depending on the oligonucleotide sequence selected, morpholinos either modify pre-mRNA splicing in the nucleus, or block translation initiation in the cytosol. After incubation for 24 hours, cells were used for RT-PCR analysis, Western blotting, cell apoptosis assays and electron microscopy analysis. Morpholino sequences were splice blocking: CGTCCTAAGAAAGAACGCACACGGA; translation blocking: ACGTAAGCTTGGAGAACATCCTG TC (http://www.gene-tools.com/).

\section{TUNEL}

Apoptotic cells were examined using the terminal dideoxynucleotidyl transferase dUTP nick end labelling (TUNEL) method with the in situ cell death detection kit, POD, according to the manufacturer's protocol (Roche, Mannheim, Germany).

\section{Electron microscopy}

Testes were fixed in glutaraldehyde for 2 hours at room temperature. Ultra-thin sections $(-90 \mathrm{~nm})$ were cut parallel to the cell monolayer and stained in uranyl acetate and lead citrate. Nuclei of spermatocytes were randomly selected using an electron microscope (JEM-1010) and capture software (SIS VELETA CCD). The observer was blinded to the genotype.

\section{Statistical analysis}

For continuous variables, differences between two groups were tested by Student t-test or t'-test (equal variances not assumed). Differences between three groups were tested by ANOVA test or Kruskal-Wallis test (equal variances not assumed). Analyses were carried out using Statistical Analysis System software (version 9.1.3; SAS Institute, Cary, NC). A two-sided $P \leq 0.05$ was considered as statistically significant.

\section{Results}

\section{FAM160B1 correlates negatively with the homozygous alternate (GG) of rs7099208.}

To investigate whether rs7099208 is associated with expression of mRNA, we analysed expression eQTL of genes surrounding rs7099208. Three genes at
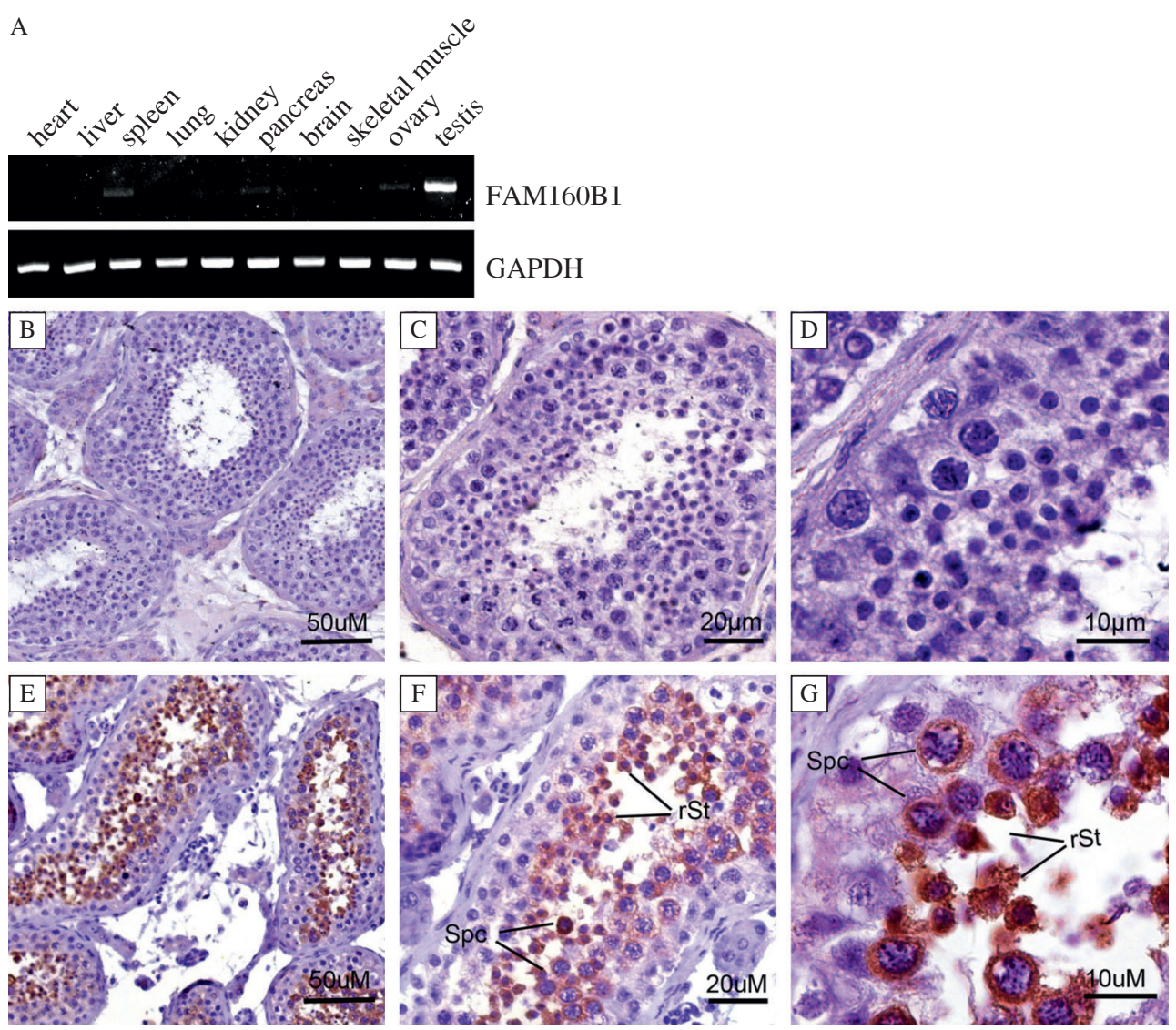

Fig. 2 FAM160B1 gene expression in Human. A: The expression of $F A M 160 B 1$ in multi-organization successively: heart, liver, spleen, lung, kidney pancreas, brain, skeletal muscle, ovarian and testes. GAPDH was used as an internal control. B-G: Immunohistochemistry of FAMI60BI in the normal Human testes. B, C, D: the negative control; E, F, G: Human testis. FAM160BI located in post-meiotic germ cells containing spermatocytes and round spermatids. 
10q25.3, FAM160B1, ABLIM1 and TRUB1, are candidates whose mRNA expression may be affected by rs7099208. We assessed the various genotypes of rs7099208 and the expression of FAMI60BI, ABLIMI and TRUBI in normal human testes, using data from the Genotype-Tissue Expression project (GTEx). The eQTL data reveal that the mean mRNA expression level of FAM160B1 in testes with the homozygous alternate (GG) genotype of rs7099208 is less than that of the homozygous reference (AA) or heterozygous (AG) genotypes (Fig. 1A). The mean expression levels of $A B L I M I$ and TRUBI were not affected by the genotype of rs7099208 (Fig. 1B and $\boldsymbol{C}$ ). In addition, the expression level of FAMI60BI in peripheral blood was not associated with the rs7099208 genotype $(P=0.8)($ Fig. 1D). These results suggest that rs7099208 is associated with mRNA expression of gene FAMI60BI in testes.

\section{FAM160B1 was predominantly expressed in human spermatocytes and round spermatids}

Using RT-PCR, expression of FAM160B1 mRNA was assessed in multiple tissue, including heart, liver, spleen, lung, kidney pancreas, brain, skeletal muscle, ovarian and testes. Expression of $F A M 160 B 1$ was predominantly observed in testes (Fig. 2A). Using immunohistochemistry, we detected FAM160B1 in male germ cells including spermatocytes and round spermatids in normal human testes (Fig. $2 \boldsymbol{E}-\boldsymbol{G}$; negative controls Fig. $2 \boldsymbol{B}-\boldsymbol{D}$ ).

A
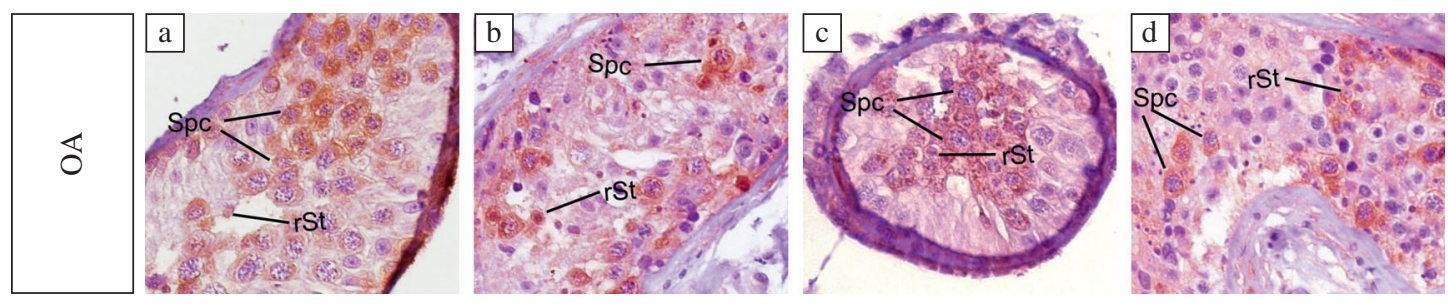

B
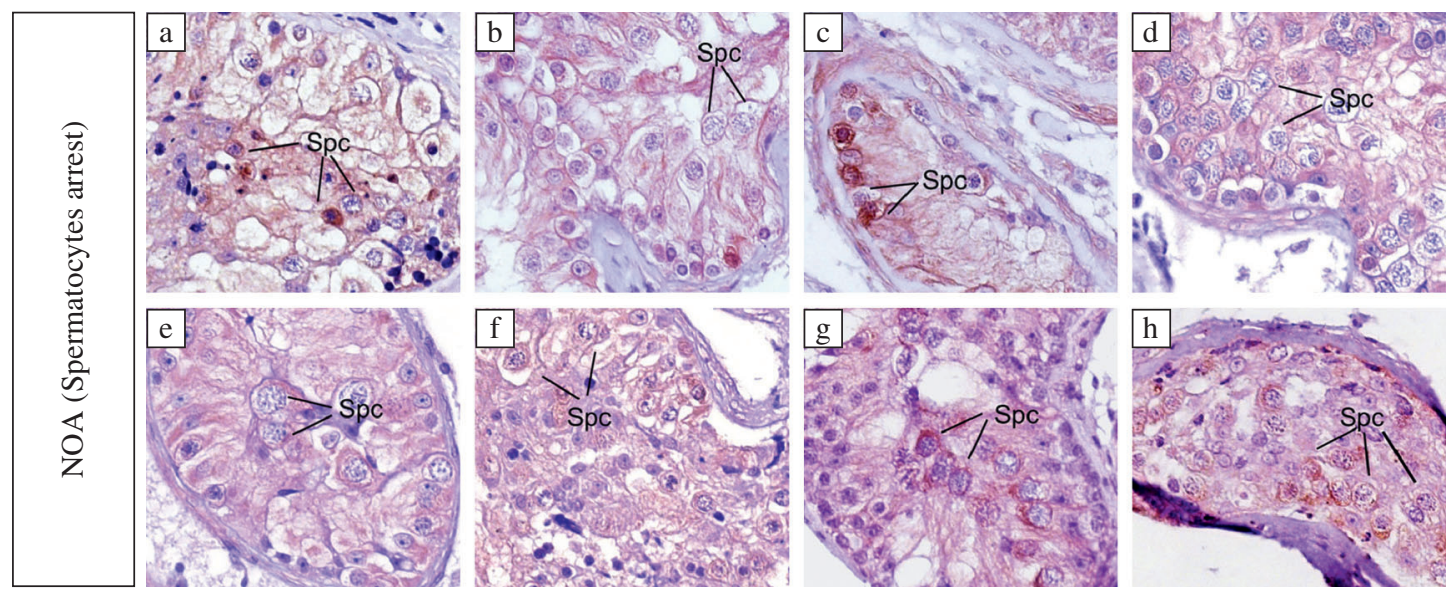

$\mathrm{C}$
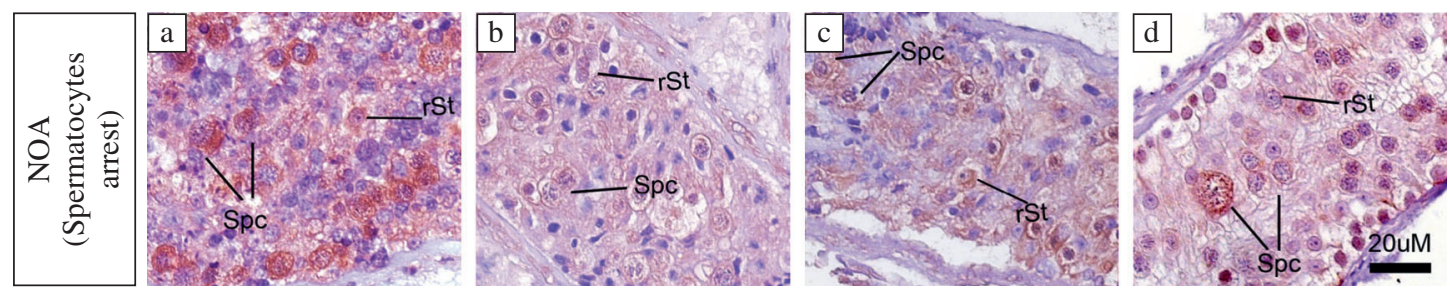

Fig. 3 Immunohistochemistry of FAM160B1 in the OA testes and NOA testes. A: a-d: 4 cases of the OA. B: in spermatocyte arrest group of NOA testes (without spermatid and mature spermatozoa but contained spermatocyte). a-h: 8 cases of the spermatocyte arrest. C: in spermatid arrest group of NOA testes (without mature spermatozoa but contained round spermatid). a-d: 4 cases of the spermatid arrest. Spc: spermatocyte, rSt: round spermatid. 
Table 1 Immunohistochemical analysis of FAM160B1 in testis section of NOA patients and control.

\begin{tabular}{lccc}
\hline & UD & Decrease & Normal \\
\hline OA & - & - & 5 \\
NOA(Spermatocyte Arrest) & 4 & 4 & 3 \\
NOA(Spermatid Arrest) & - & 3 & 3 \\
\hline
\end{tabular}

*UD: Undetectable

\section{Expression of FAM160B1 was reduced or undetectable in testes from NOA patients}

Then, we examined FAM160B1 expression in patients within NOA and OA testes. Arrest of spermatocytes and spermatids are common phenotypes of NOA. Our NOA samples were sorted to the spermatocyte arrest group (without spermatid and mature spermatozoa but contained spermatocyte) and spermatid arrest group (without mature spermatozoa but contained round spermatid). For all five patients with OA,
FAM160B1 was detected in spermatocytes and round spermatids $(\boldsymbol{F i g}$. 3A $)$. Immunohistochemical analysis of 11 cases of spermatocyte arrest testes $(\boldsymbol{F i g}$. $3 \boldsymbol{B})$ revealed that only three of 11 showed normal positive FAM160B1 signals in spermatocyte (Fig. $3 \boldsymbol{B} \boldsymbol{g}, \boldsymbol{h}$ and $\boldsymbol{j})$, and the FAM160B1 signal in spermatocyte was undetectable in four cases (Fig. $3 \boldsymbol{B} \boldsymbol{b}, \boldsymbol{d}, \boldsymbol{e}$ and $\boldsymbol{k}$ ). On the other hand, in spermatocyte and round spermatid (Fig. 3C), three in six cases of spermatid arrest testes showed normal positive FAM160B1 signals (Fig. 3C a, $\boldsymbol{d}$ and $\boldsymbol{f}$ ). In the remaining seven NOA samples, including spermatocytes and round spermatids arrest patients (Fig. $3 \boldsymbol{B} \boldsymbol{c}, \boldsymbol{f}$ and $\boldsymbol{i}$; Fig. $3 \boldsymbol{C} \boldsymbol{b}, \boldsymbol{c}$ and $\boldsymbol{e}), F A M 160 B 1$ signals were decreased relative to controls. These results suggest that FAM160B1 expression is associated with NOA defects (Table 1).

\section{Analysis of $F A M 160 B 1$ in mouse germ cells}

In mice, the expression of FAM160B1 mRNA was assessed in multiple tissue types and found predominantly
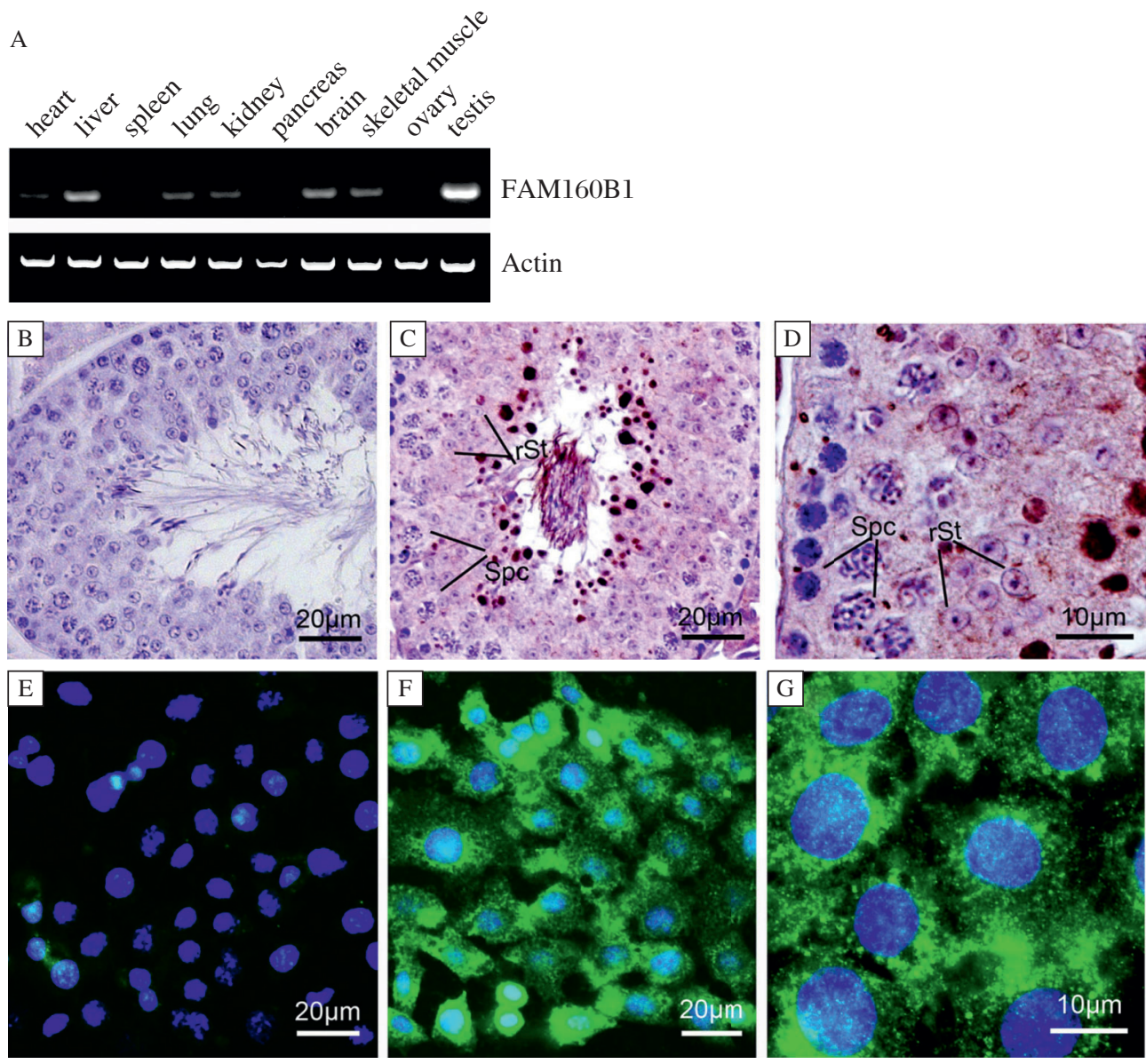

Fig. 4 FAM160B1 gene expression in Mice. A: The expression of FAM160B1 in multi-organization successively: heart, liver, spleen, lung, kidney pancreas, brain, skeletal muscle, ovarian and testes. Actin was used as an internal control. B-D: immunohistochemistry of FAM160B1 in the mice testes. B: the negative control. C, D: FAM160B1 showed punctate signals in germ cells including spermatocytes (Spc) and round sperm (rSt), and highly expressed in elongated sperm. E-G: Expression of FAM160B1 in GC2 cells. E: the negative control. F, G: FAM160B1 located in the cytoplasm and part of the nucleus. 
in testes as the same within human (Fig. 4A). Using immunohistochemistry, FAMI60BI was detected in spermatocytes and the later stage (Fig. 4C, D; Supplementary Figure, which is available online; negative control in Fig. $4 B$ ). To investigate the biological role of $F A M 160 B 1$ in germ cells in vitro, murine germ cell line $\mathrm{GC} 2$ was used. Immunofluorescence of FAM160B1 located to the cytoplasm and part of the nucleus in cell line GC2 (Fig. 4F, G; negative control Fig. 4E).
Two morpholinos (splice blocking and translation blocking) were used to knockdown expression of FAM160B1 in GC2 cells. Following incubation with $10 \mu \mathrm{M}$ splice blocking morpholinos for 24 hours, the number of GC2 cells decreased (Fig. 5A, B and $\boldsymbol{C}$ ). Knockdown efficiency of splice blocking reached approximately $90 \%$ at the mRNA level (Fig. 5D). Similar results were observed after knockdown with translation blocking morpholinos (Fig. $5 \boldsymbol{E}, \boldsymbol{F}$ and

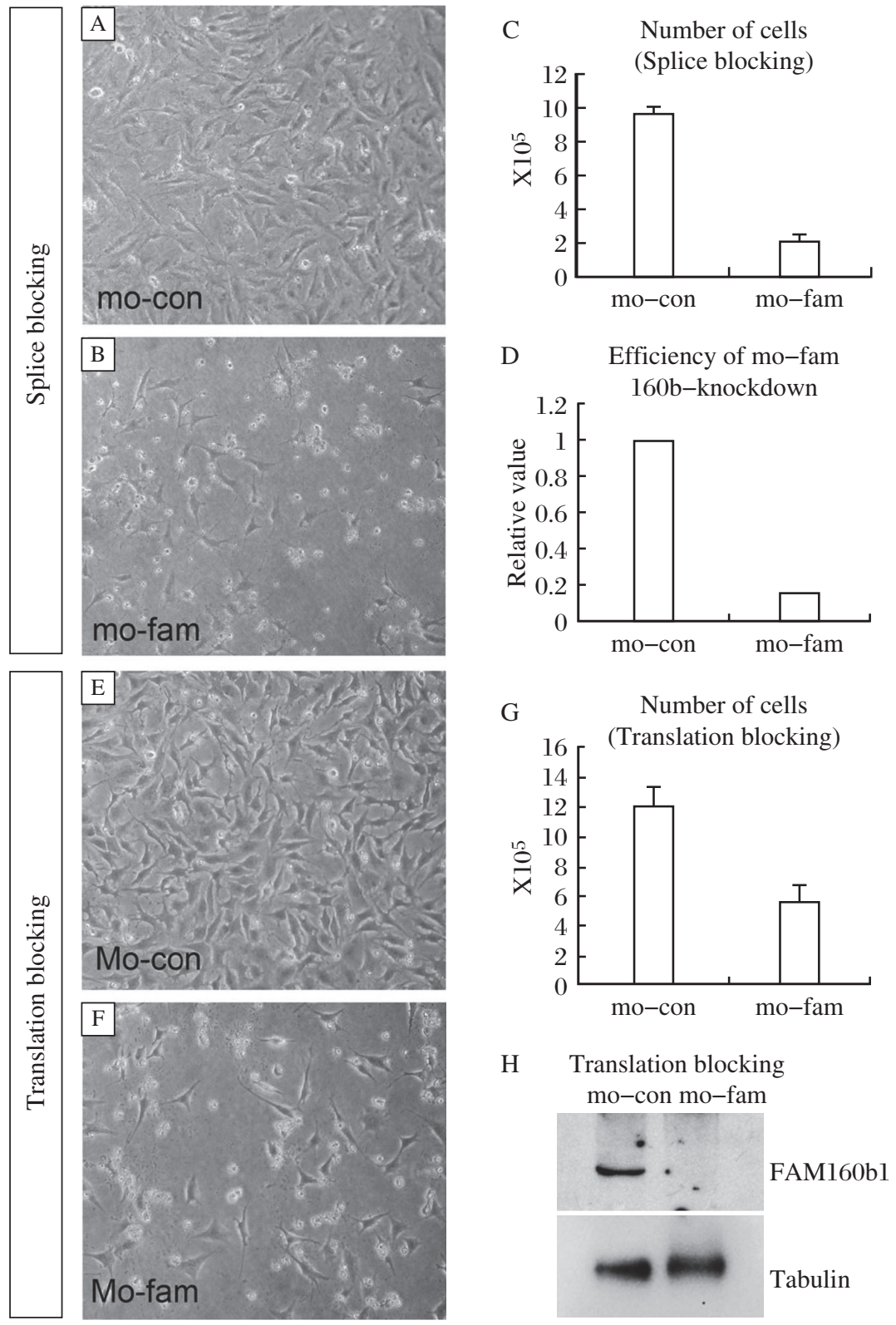

Fig. 5 FAM160B1 was knocked down in murine germ cell lines GC2 with morpholinos. A, B: knockdown with splice blocking morpholinos; C: cell counting experiments between control (mo-con) and $F A M 160 B 1$ (mo-fam). ${ }^{*} P<0.01$. D: Verification of the efficiency of knockdown after morpholinos blocking. Actin was used as an internal control. $* * P<0.01$. E, F: knockdown with translation blocking morpholinos. G: cell counting experiments between control (Mo-con) and FAM160B1 (Mo-fam). $* * P<0.01$. H: Verification of the efficiency of knockdown after morpholinos blocking. Tubulin was used as an internal control. 


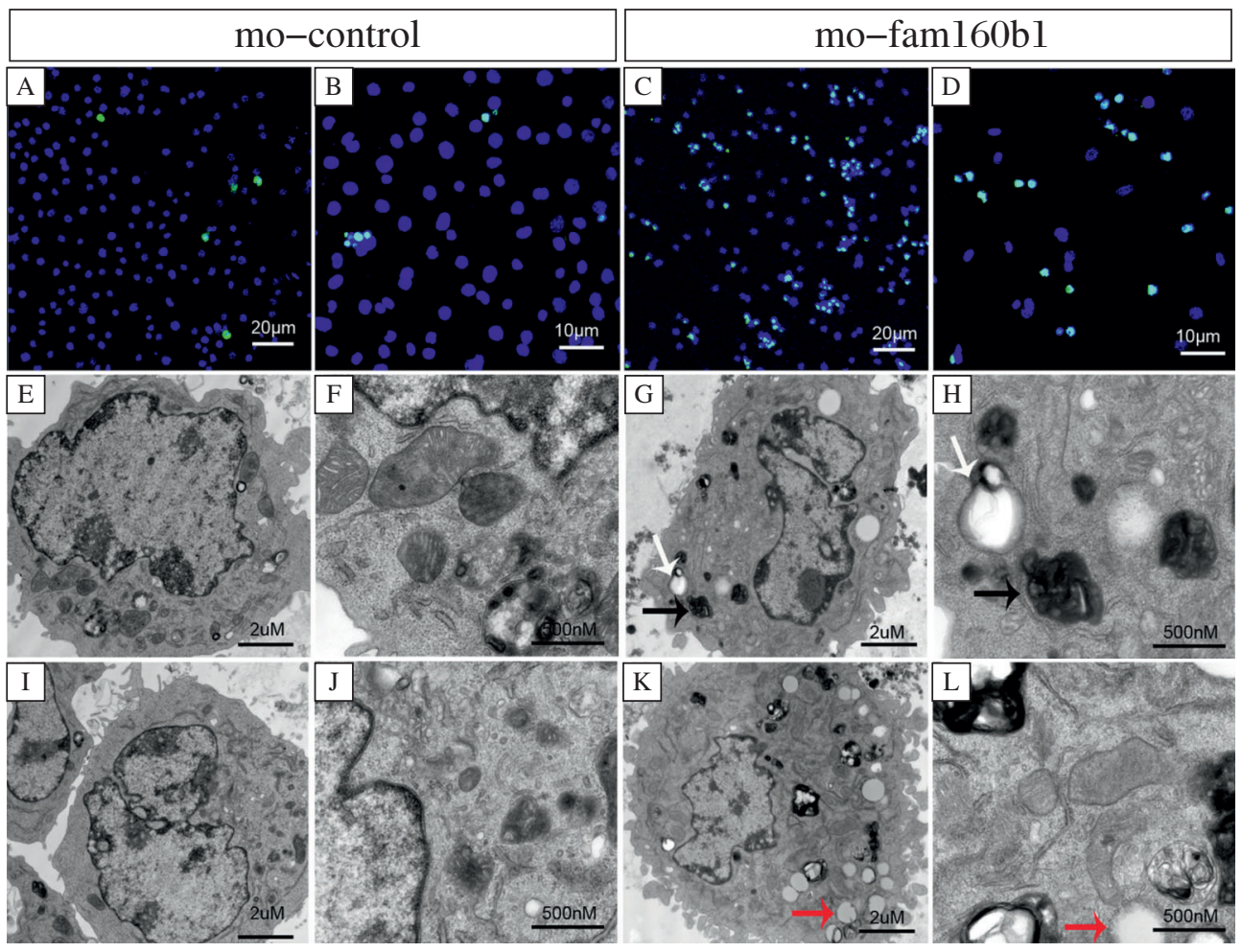

Fig. 6 TUNEL analysis and electron microscopy after transfection with morpholinos specific to FAM160B1. A, C: Positive signals mo-control and mo-FAM160B1 with TUNEL analysis. B, D: Larger image of A, B. E-L: Electron microscopy under morpholinos inhibiting of the two groups. E, I: the control, F, J: Larger image of E, I. G, K: in perinuclear of cells there were more lipofuscin (black arrow), lipid droplets (red arrow), vacuoles (white arrow), etc. H, L: Larger image of G, K.

$\boldsymbol{G})$. Translation blocking of FAMI60B1 also showed high efficiency (Fig. 5H).

TUNEL analysis was performed 24 hours after knockdown with morpholinos. Positive TUNEL signals increased significantly (Fig. $6 \boldsymbol{A}-\boldsymbol{D})$. Using electron microscopy, we found an accumulation of lipofuscin, lipid droplets and vacuoles in the perinuclear space of GC2 cells after inhibiting FAM160B1 (Fig. 6E-L). These results suggest that FAM160B1 knockdown induced murine germ cell apoptosis.

\section{Discussion}

Only a few cases of NOA are caused by gene mutations, for example, seipin mutation ${ }^{[14]}$. Due to the high incidence of both NOA and SNPs, it is reasonable to hypothesise that the main genetic cause of NOA is the collaborative effect of SNPs. In recent years, susceptibility loci associated with multiple diseases have been identified in GWAS, including breast cancer ${ }^{[15-16]}$, colorectal cancer ${ }^{[17-18]}$, endometrial cancer ${ }^{[19]}$, follicular lymphoma $^{[20]}$, gastric cancer ${ }^{[21]}$, hepatocellular carcinoma ${ }^{[22-23]}$, Hodgkin's lymphoma ${ }^{[24]}$, lung cancer $^{[25]}$, melanoma ${ }^{[26]}$, nasopharyngeal carcinoma ${ }^{[27]}$, neuroblastoma ${ }^{[28]}$, ovarian cancer ${ }^{[5,29]}$, pancreatic cancer ${ }^{[30-31]}$, prostate cancer $^{[32-37]}$, renal cell carcinoma ${ }^{[38]}$, thyroid cancer $^{[39]}$, urinary bladder cancer ${ }^{[4]}$ and congenital heart malformations ${ }^{[40]}$. In our previous study, NOA susceptibility loci were identified based on GWAS data from thousands of patients ${ }^{[8-9]}$. However, the mechanisms of disease remained unclear. Here, we analysed eQTL of genes in the region of an NOA susceptibility locus and used the data arising to identify the disease mechanism. The success of our approach suggests that GWAS could be a key to efficient characterisation of gene variations involved in NOA. Recently, we discovered several potential protein markers for cancers using GWAS analysis $^{[6-7]}$.

Our aim of this study is to investigate the relationship between a NOA susceptibility locus rs7099208 and genes near this region, like FAM160B1. Based on eQTL data from GTEx, we found that rs7099208 has a mild effect on expression of FAM160B1. Moreover, we demonstrated that $F A M 160 B 1$ is essential for germ cell survival and reduce expression in germ cells of some NOA patients. Our study makes a connection between a mild effect of SNP (from GWAS, like rs7099208) and spermatogenic gene (like FAM160B1). It should be noticed that common SNPs (MAF $>1 \%$ ) widely exist in both patients and normal population. Mild effect may not cause a huge 
change of single gene expression, but combination of mild effects from several SNPs could contribute a severe consequence and lead to a disease (like NOA).

Previous studies have shown that in NOA patients minor allele frequency (MAF) of rs7099208 is higher than the control population. Mild effect of rs7099208 may not cause a dramatic change of FAM160B1 because variant homozygote of rs7099208 exists in normal population. Although the mechanism of regulation of FAM160B1 is unclear, lack of FAM160B1 leads to germ cell apoptosis. We found the expression of FAM160B1 is changed in NOA patients. We concluded that variant homozygote of rs7099208 caused mild effect on spermatogenic gene FAM160B1.

Unlike severe effect caused by gene mutation, common SNPs affect gene expression may not be contributed by singe SNP variant but combinations of mild effect of SNPs. The functional combinations of SNPs are still under discovery. Our data provide a new way to investigate spermatogenesis associated SNPs. The full understanding of FAMI60BI in spermatogenesis would be worthy to investigate in further studies.

\section{Acknowledgment}

This work was supported by the grants from the 973 program (2011CB944304 and 2015CB943003).

\section{References}

[1] Sharpe RM. Sperm counts and fertility in men: a rocky road ahead. Science \& Society Series on Sex and Science[J]. EMBO Reports, 2012,13(5):398-403.

[2] Maduro MR, Lamb DJ. Understanding new genetics of male infertility[J]. J Urol, 2002,168(5):2197-2205.

[3] Ferlin A, Moro E, Garolla A, et al. Human male infertility and Y chromosome deletions: role of the AZF-candidate genes DAZ, RBM and DFFRY[J]. Hum Reprod, 1999, 14(7):1710-1716.

[4] Wu X, Ye Y, Kiemeney LA, et al. Genetic variation in the prostate stem cell antigen gene PSCA confers susceptibility to urinary bladder cancer[J]. Nat Genet, 2009,41(9): 991995.

[5] Goode EL, Chenevix-Trench G, Song H, et al. A genomewide association study identifies susceptibility loci for ovarian cancer at 2q31 and 8q24[J]. Nat Genet, 2010,42(10): 874-879.

[6] Liu M, Chen J, Hu L, et al. HORMAD2/CT46.2, a novel cancer/testis gene, is ectopically expressed in lung cancer tissues[J]. Mol Hum Reprod, 2012,18(12):599-604.

[7] Liu M, Hu Z, Qi L, et al. Scanning of novel cancer/testis proteins by human testis proteomic analysis[J]. Proteomics, 2013,13(7):1200-1210.

[8] Hu Z, Xia Y, Guo X, et al. A genome-wide association study in Chinese men identifies three risk loci for nonobstructive azoospermia[J]. Nat Genet, 2012,44(2): 183-186.
[9] $\mathrm{Hu} \mathrm{Z,} \mathrm{Li} \mathrm{Z,} \mathrm{Yu} \mathrm{J,} \mathrm{et} \mathrm{al.} \mathrm{Association} \mathrm{analysis} \mathrm{identifies} \mathrm{new}$ risk loci for non-obstructive azoospermia in Chinese men[J]. Nat Commun, 2014,5:3857.

[10] The Genotype-Tissue Expression (GTEx) project[J]. Nat Genet. 2013,45(6):580-585.

[11] Liu M, Hales BF, Robaire B. Effects of four chemotherapeutic agents, bleomycin, etoposide, cisplatin, and cyclophosphamide, on DNA damage and telomeres in a mouse spermatogonial cell line[J]. Biol Reprod. 2014,90(4):72.

[12] Liu M, Shi X, Bi Y, et al. SHCBP1L, a conserved protein in mammals, is predominantly expressed in male germ cells and maintains spindle stability during meiosis in testis[J]. Mol Hum Reprod. 2014,20(6):463-475.

[13] Liu M, Qi L, Zeng Y, et al. Transient scrotal hyperthermia induces lipid droplet accumulation and reveals a different ADFP expression pattern between the testes and liver in mice[J]. PLoS One. 2012,7(10):e45694.

[14] Jiang M, Gao M, Wu C, et al. Lack of testicular seipin causes teratozoospermia syndrome in men[J]. Proc Natl Acad Sci U S A. 2014,111(19):7054-7059.

[15] Thomas G, Jacobs KB, Kraft P, et al. A multistage genomewide association study in breast cancer identifies two new risk alleles at 1p11.2 and 14q24.1 (RAD51L1)[J]. Nat Genet, 2009,41(5):579-584.

[16] Turnbull C, Ahmed S, Morrison J, et al. Genome-wide association study identifies five new breast cancer susceptibility loci[J]. Nat Genet. 2010,42(6):504-507.

[17] Tomlinson IP, Webb E, Carvajal-Carmona L, et al. A genome-wide association study identifies colorectal cancer susceptibility loci on chromosomes 10p14 and 8q23.3[J]. Nat Genet, 2008,40(5):623-630.

[18] Houlston RS, Cheadle J, Dobbins SE, et al. Meta-analysis of three genome-wide association studies identifies susceptibility loci for colorectal cancer at 1q41, 3q26.2, 12q13.13 and 20q13.33[J]. Nat Genet, 2010,42(11):973-977.

[19] Spurdle AB, Thompson DJ, Ahmed S, et al. Genomewide association study identifies a common variant associated with risk of endometrial cancer[J]. Nat Genet, 2011,43(5):451-454.

[20] Conde L, Halperin E, Akers NK, et al. Genome-wide association study of follicular lymphoma identifies a risk locus at 6p21.32[J]. Nat Genet, 2010,42(8):661-664.

[21] Shi Y, Hu Z, Wu C, et al. A genome-wide association study identifies new susceptibility loci for non-cardia gastric cancer at 3q13.31 and 5p13.1[J]. Nat Genet, 2011,43(12): 1215-1218.

[22] Zhang H, Zhai Y, Hu Z, et al. Genome-wide association study identifies 1 p36.22 as a new susceptibility locus for hepatocellular carcinoma in chronic hepatitis B virus carriers[J]. Nat Genet, 2010,42(9):755-758.

[23] Kumar V, Kato N, Urabe Y, et al. Genome-wide association study identifies a susceptibility locus for HCV-induced hepatocellular carcinoma[J]. Nat Genet, 2011,43(5):455-458.

[24] Enciso-Mora V, Broderick P, Ma Y, et al. A genome-wide association study of Hodgkin's lymphoma identifies new susceptibility loci at 2p16.1 (REL), 8q24.21 and 10p14 (GATA3)[J]. Nat Genet, 2010,42(12):1126-1130.

[25] Hu Z, Wu C, Shi Y, et al. A genome-wide association study identifies two new lung cancer susceptibility loci at $13 \mathrm{q} 12.12$ and $22 \mathrm{q} 12.2$ in Han Chinese[J]. Nat Genet, 2011,43(8):792-796. 
[26] Bishop DT, Demenais F, Iles MM, et al. Genome-wide association study identifies three loci associated with melanoma risk[J]. Nat Genet, 2009,41(8): 920-925.

[27] Bei JX, Li Y, Jia WH, et al. A genome-wide association study of nasopharyngeal carcinoma identifies three new susceptibility loci[J]. Nat Genet, 2010,42(7):599-603.

[28] Capasso M, Devoto M, Hou C, et al. Common variations in BARD1 influence susceptibility to high-risk neuroblastoma[J]. Nat Genet, 2009,41(6):718-723.

[29] Bolton KL, Tyrer J, Song H, et al. Common variants at 19 p13 are associated with susceptibility to ovarian cancer[J]. Nat Genet, 2010,42(10):880-884.

[30] Petersen GM, Amundadottir L, Fuchs CS, et al. A genome-wide association study identifies pancreatic cancer susceptibility loci on chromosomes 13q22.1, 1q32.1 and 5p15.33[J]. Nat Genet, 2010,42(3):224-228.

[31] Amundadottir L, Kraft P, Stolzenberg-Solomon RZ, et al. Genome-wide association study identifies variants in the ABO locus associated with susceptibility to pancreatic cancer[J]. Nat Genet, 2009,41(9):986-990.

[32] Gudmundsson J, Sulem P, Gudbjartsson DF, et al. Genomewide association and replication studies identify four variants associated with prostate cancer susceptibility[J]. Nat Genet, 2009,41(10):1122-1126.

[33] Haiman CA, Chen GK, Blot WJ, et al. Genome-wide association study of prostate cancer in men of African ancestry identifies a susceptibility locus at $17 \mathrm{q} 21[\mathrm{~J}]$. Nat Genet, 2011,43(6):570-573.

[34] Eeles RA, Kote-Jarai Z, Al Olama AA, et al. Identification of seven new prostate cancer susceptibility loci through a genome-wide association study[J]. Nat Genet, 2009,41(10):1116-1121.

[35] Thomas G, Jacobs KB, Yeager M, et al. Multiple loci identified in a genome-wide association study of prostate cancer[J]. Nat Genet, 2008,40(3):310-315.

[36] Yeager M, Chatterjee N, Ciampa J, et al. Identification of a new prostate cancer susceptibility locus on chromosome 8q24[J]. Nat Genet, 2009,41(10):1055-1057.

[37] Eeles RA, Kote-Jarai Z, Giles GG, et al. Multiple newly identified loci associated with prostate cancer susceptibility[J]. Nat Genet, 2008,40(3):316-321.

[38] Purdue MP, Johansson M, Zelenika D, et al. Genomewide association study of renal cell carcinoma identifies two susceptibility loci on 2p21 and 1lq13.3[J]. Nat Genet, 2011,43(1):60-65.

[39] Gudmundsson J, Sulem P, Gudbjartsson DF, et al. Common variants on $9 \mathrm{q} 22.33$ and $14 \mathrm{q} 13.3$ predispose to thyroid cancer in European populations[J]. Nat Genet, 2009,41(4):460-464.

[40] Hu Z, Shi Y, Mo X, et al. A genome-wide association study identifies two risk loci for congenital heart malformations in Han Chinese populations[J]. Nat Genet, 2013,45(7):818-821.

\section{CLINICAL TRIAL REGISTRATION}

The Journal requires investigators to register their clinical trials in a public trials registry for publication of reports of clinical trials in the Journal. Information on requirements and acceptable registries is available at www.icmje.org/faq_clinical.html. 